\title{
GLAD!
}

Revue sur le langage, le genre, les sexualités

05 | 2018

Raconter les sexualités depuis la marge

\section{L'inceste et le droit pénal français contemporain : lorsque l'application du droit dans les tribunaux révèle une indicible frontière}

Incest and the Contemporary French Penal Code: When Judicial Practices in

Criminal Courts Reveal an Unspeakable Border

\section{Marie Romero}

\section{OpenEdition}

Journals

Édition électronique

URL : http://journals.openedition.org/glad/1230

ISSN : 2551-0819

Éditeur

Association GSL

Référence électronique

Marie Romero, «L'inceste et le droit pénal français contemporain : lorsque l'application du droit dans les tribunaux révèle une indicible frontière », GLAD! [En ligne], 05 | 2018, mis en ligne le 15 décembre 2018, consulté le 17 décembre 2020. URL : http://journals.openedition.org/glad/1230

Ce document a été généré automatiquement le 17 décembre 2020.

\section{(c) $(1)$}

La revue GLAD! est mise à disposition selon les termes de la Licence Creative Commons Attribution Pas d'Utilisation Commerciale - Pas de Modification 4.0 International. 


\title{
L'inceste et le droit pénal français contemporain : lorsque l'application du droit dans les tribunaux révèle une indicible frontière
}

\author{
Incest and the Contemporary French Penal Code: When Judicial Practices in \\ Criminal Courts Reveal an Unspeakable Border
}

Marie Romero

\section{Introduction}

1 Il aura fallu plus de deux siècles et une véritable transformation morale pour considérer les violences sexuelles sur mineurs comme crime ultime d'une société, comme l'a souligné l'historienne Anne-Claude Ambroise-Rendu dans son ouvrage de référence, Histoire de la pédophilie. XIXe-XXe siècle: «Chacun voit désormais dans les attouchements sexuels pratiqués par un adulte sur un enfant une transgression majeure, un des pires crimes ${ }^{1}$. C'est dans ce contexte de nouvelles sensibilités aux violences sexuelles sur mineurs, d'attention croissante à la souffrance des victimes et aux conséquences post-traumatiques, qu'a émergé la question de la répression de l'inceste. Elle a été au cœur des politiques législatives à partir du milieu des années 2000 en France : l'urgence a alors été de sortir du tabou sociétal sur l'inceste et de rendre plus visible un phénomène trop longtemps occulté par le législateur².

2 Cependant, à la différence d'autres législations européennes (Allemagne, Pays-Bas, Angleterre, Danemark, et Suisse), l'inceste n'est pas une infraction spécifique dans le droit pénal français ${ }^{3}$. Il n'est pas réprimé en tant que tel, et contrairement aux préconisations législatives européennes ${ }^{4}$, il reste indirectement visé, par la circonstance aggravante du lien d'ascendance ou d'autorité avec la victime, dans le viol, l'agression sexuelle et l'atteinte sexuelle sur mineur de 15 ans. Si l'ancien droit en avait 
fait un crime spécifique (Vigarello, $1998^{5}$; Giulianni, 20106), aujourd'hui c'est sous les auspices du viol qu'il est le plus sévèrement condamné 7 . Cette particularité du droit pénal français, de ne viser qu'indirectement l'inceste sans le nommer explicitement, a suscité de vifs débats à la fin des années 2000, entre pénalistes, professionnels de justice et associations de victimes.

3 L'enjeu a été d'une part, de savoir comment traduire juridiquement une notion anthropologique complexe, et d'autre part, s'il fallait en faire une infraction autonome pour sortir de la délicate question de preuve du non-consentement du mineur. Or, dans la loi n²010-121 du 8 février 2010 sur l'inceste, le législateur n'a pas fait de l'inceste une infraction spécifique, mais simplement une surqualification incestueuse ${ }^{8}$ s'ajoutant aux infractions déjà existantes de viol, d'agression sexuelle et d'atteinte sexuelle. Les articles 222-31-1 et 227-27-2 du Code pénal, visant cette surqualification, ont fait l'objet de deux questions prioritaires de constitutionnalité du fait de l'imprécision du périmètre familial. Ils ont été abrogés par le conseil constitutionnel ${ }^{9}$ et réintroduits dans la loi n ${ }^{\circ}$ 2016-297 du 14 mars 2016 selon une liste énumérative de personnes ${ }^{10}$.

4 En dépit de ces avancées, l'ensemble de ces règles a fait l'objet de vives critiques par la doctrine. D'abord parce qu'elles sont sans conséquence sur le plan répressif, la qualification pénale d'inceste n'apportant qu'un intérêt «symbolique » et non une peine supplémentaire par rapport à la circonstance aggravante qu'a toujours constitué l'inceste. Ensuite parce que ces nouvelles dispositions ne s'appliquent qu'aux mineurs et ne prennent pas en compte les victimes d'inceste majeures. Ainsi des viols commis par un père sur sa fille de 16 ans pourront être qualifiés d'« incestueux » mais pas ceux commis sur sa fille de 18 ans. Cette exclusion des victimes d'inceste majeures ${ }^{11}$ est en totale contradiction avec la finalité de la loi « qui était de prendre en considération la particularité de la souffrance des victimes d'actes incestueux $»^{12}$, comme l'a rappelé, également, la sociologue Sylvie Cromer dans le rapport d'expertise sur les violences sexuelles à caractère incestueux ${ }^{13}$. Pourquoi toutes les victimes d'inceste ne bénéficient pas de la même protection juridique ? Pourquoi une focalisation sur les seules victimes d'inceste mineures? Est-ce par ce que les mineurs sont considérées "comme les victimes les plus vulnérables entre toutes ${ }^{14}$, au-delà même de la question de l'inceste ?

$5 \mathrm{Au}$ final, la qualification pénale de l'inceste apparaît complexe et contradictoire car indissociable de la question de la minorité des victimes. Face à l'intolérance croissante de la société à l'égard de toute sexualité imposée par un adulte aux mineurs, les conséquences de ces changements sont importantes dans la recomposition des normes du permis et de l'interdit sexuel autour des asymétries d'âge (mineur/majeur). Ce qui est interdit moralement et juridiquement c'est pour un majeur d'avoir une relation sexuelle avec un mineur parce qu'il s'agit d'un être extrêmement vulnérable et en devenir, qui porte quelque chose de sacré dans nos sociétés. Aussi, dans cet article, je tenterai d'interroger la singularité de la qualification pénale de l'inceste et les pratiques judiciaires à l'œuvre à l'échelle de quatre tribunaux observés dans le cadre d'une thèse en cours. Je montrerai d'abord la façon dont le droit a fait de l'inceste un cas particulier des infractions sexuelles et ensuite les répercussions sur l'appréhension pénale du non-consentement du mineur. L'hypothèse centrale étant que les normes pénales sont redéfinies et la ligne de démarcation entre les âges devient l'enjeu majeur autour duquel se réorganise la qualification juridique. 


\section{L'inceste, un cas particulier des infractions sexuelles, au sein desquelles prévaut l'atteinte au consentement}

6 Le droit pénal a fait de l'inceste un cas particulier des infractions sexuelles puisqu'il est indirectement sanctionné à partir de la circonstance aggravante d'ascendant ou d'autorité sur la victime. Ainsi, selon la règle d'incrimination pénale de l'inceste, le lien de famille (ascendant ou autorité) est un critère qui aggrave certaines infractions (viol, agression sexuelle, atteinte sexuelle sur mineur de 15 ans) ou qui en constitue d'autres (atteinte sexuelle sur mineur de plus de 15 ans $^{15}$ ). Le viol par ascendant constitue la forme d'inceste la plus grave et la plus sévèrement punie puisqu'il fait encourir 20 ans de réclusion criminelle ${ }^{16}$. Dans les autres infractions, le cumul des deux critères (lien de famille et âge) élève les peines à 10 ans d'emprisonnement pour l'agression sexuell ${ }^{17}$ et pour l'atteinte sexuelle sur mineur de 15 ans $^{18}$. L'âge est donc un critère déterminant la gravité des peines lorsqu'il se conjugue avec le lien de famille dans l'infraction. La sévérité est la même dans un cas et dans l'autre. C'est désormais sous ses deux aspects, lien de famille et âge de la victime, que l'inceste est appréhendé par le droit pénal français.

7 Cette évolution découle des nouvelles logiques d'incrimination pénale voulues par le législateur. À la différence de l'ancien code qui visait le lien de famille au sein d'un seul article de la même section ${ }^{19}$, le nouveau Code pénal de $1994^{20}$ l'a introduit dans deux sections complètement distinctes: celle des atteintes à l'intégrité physique et psychique de la personne et celle des atteintes au mineur et à sa famille. Le législateur entend ainsi protéger tout ce qui touche au défaut de consentement d'un côté et de l'autre à la vulnérabilité du mineur. Ce faisant, il a défini les atteintes sexuelles sur mineurs, à l'opposé des agressions sexuelles, c'est-à-dire sans «violence, contrainte, menace ou surprise». Ce qui signifie qu'il n'est pas besoin de ces modalités «situationnelles» pour caractériser l'infraction envers un mineur, car celui-ci est supposé ne jamais pouvoir consentir véritablement librement.

8 Le législateur a donc établi une ligne de démarcation entre les relations sexuelles qui sont interdites parce qu'elles portent atteinte au consentement de la personne (section des agressions sexuelles) et celles prohibées parce que le consentement du mineur n'est statutairement pas valable (section de la mise en péril du mineur). Ce changement ne peut se comprendre qu'à l'aune de la nouvelle référence au consentement devenu la summa diviso des infractions sexuelles. L'atteinte au consentement comme matrice juridique du nouveau régime infractionnel n'est ni inscrite en tant que telle dans la loi, ni définie juridiquement, mais découle implicitement des éléments constitutifs des agressions sexuelles qui se trouvent à l'article 222-22 du Code pénal : «avec violence, contrainte, menace ou surprise ». Elle est appréhendée différemment selon qu'il s'agit de réprimer l'agression sexuelle ou l'atteinte sexuelle. Sociologiquement, l'atteinte au consentement a deux sens: un non-consentement situationnel qui se déduit de la violence, contrainte, menace ou surprise des circonstances de l'acte et un nonconsentement statutaire qui se déduit de l'âge du mineur et consiste plus précisément en une incapacité de consentir librement (un mineur de moins de 15 ans est supposé ne jamais pouvoir consentir librement à un adulte).

9 Partant de la thèse des trois révolutions du consentement d'Irène Théry (2002), nous postulons que, dans ce nouvel ordre du permis et de l'interdit sexuel qui se focalise désormais sur le consentement et son contraire, le viol, la place de l'inceste est devenue 
de plus en plus complexe. L'inceste ne fait plus le cœur de l'interdit sexuel au sens de la dimension la plus haute, comme l'explique Irène Théry, «ce que nous combattons désormais sous le nom d'inceste, c'est moins l'interdit des places distinguant dans un système symbolique alliance et filiation (on constate aisément que personne ne connaît avec précision le cercle exact des prohibitions matrimoniales) que la forme la plus grave de ce qui est désormais le nouveau cœur de l'interdit: le viol $»^{21}$. Ces questions convergent vers celle du non-consentement de la victime. Il est devenu en droit l'élément déterminant des infractions d'agressions sexuelles, fondé sur les éléments contextuels des faits : la violence, la contrainte, la menace, la surprise. Toute l'attention est donc portée sur les circonstances de la relation sexuelle en cause : qui, quand, comment, de quelle manière, pourquoi, autrement dit, ce que nous avons nommé le non-consentement situationnel.

Cependant, dans la pratique judiciaire la question du consentement engage des questions de preuves extrêmement complexes, en particulier avec les mineurs victimes d'inceste. Les juges sont tenus de rechercher au cas par cas, si le mineur victime a été forcé, et ce faisant, à caractériser en quoi les actes incestueux ont été commis par "violence, menace, contrainte, ou surprise ». Or, il peut paraître incongru de se poser la question de la preuve du non-consentement du mineur victime d'inceste. Comment en effet prouver l'absence de consentement du mineur lorsque celui-ci n'a pas résisté ou n'a pas été frappé, lorsque son état de dépendance affective l'a empêché de s'opposer à son agresseur et l'a conduit à se résigner, à subir en silence les actes incestueux (Vrignaux, 1994; Porchy, 200322)? Comment prendre en compte la spécificité du «fait d'inceste » (Glandier-Lescure, 2006) au sein de la sphère judiciaire ?

11 Afin d'assouplir ces difficultés en matière probatoire, le législateur a décidé, en 2010, d'étendre la notion de contrainte morale à l'écart d'âge entre mineur victime et auteur et au lien d'autorité de ce dernier ${ }^{23}$. Mais cette disposition est demeurée très controversée par la doctrine. Certains pénalistes ont estimé que la règle est « incomplète, car en faisant référence à la seule "autorité", elle ne permet pas de démontrer la contrainte imposée par un membre de la famille qui n'en bénéficie pas, alors même que l'inceste se définit en priorité par ce rapport $»^{24}$. Pour d'autres, la question de l'âge a été au cœur des débats. Selon une jurisprudence en cours ${ }^{25}$, le très jeune âge de la victime peut constituer une contrainte : les enfants de moins de 6 ans, du fait de leur manque de discernement, sont considérés comme étant « incapable de réaliser la nature et la gravité des actes qui leur sont imposés $»^{26}$. Nombre de pénalistes ont vivement critiqué l'absence d'âge légal de non-consentement dans la loi et estimé que celle-ci devait changer (Koering-Joulin, 2006; Guéry, 2010 ; Delors, 2011). Mais comme il a été rappelé dans une décision commentée du Conseil constitutionnel, « il appartient aux juridictions d'apprécier si le mineur était en état de consentir à la relation sexuelle en cause $\aleph^{27}$.

12 Les débats se sont poursuivis, à l'occasion de deux affaires fortement médiatisées en France fin $2017^{28}$, entre associations de victimes et de protection de l'enfance, pénalistes, professionnels de justice et politiques, autour d'un projet de loi sur les violences sexuelles et sexistes. L'un des enjeux a été de savoir s'il fallait changer la loi et faire entrer un âge légal de non-consentement du mineur victime pour sortir de ce vaste débat sur la preuve (le très controversé article 2). Dans ce cadre, certains parlementaires ont proposé de retenir l'âge de 18 ans, d'autres 16 ans $^{29}$ pour les mineurs victimes d'inceste. Cependant, par crainte d'une censure par le conseil 
constitutionnel, et contrairement à ce qui avait été annoncé par le gouvernement, le législateur n'a pas fait entrer un âge légal de non-consentement du mineur. Ainsi, la loi n²018-703 sur les violences sexuelles et sexistes, définitivement adoptée le 3 août 2018, a étendu la notion juridique de contrainte à l'« abus de vulnérabilité » du mineur de moins de $15 \mathrm{ans}^{30}$. Le qualificatif « incestueux » a été élargi aux infractions commises sur des victimes majeures ${ }^{31}$, et les délais de prescription ont été allongés de 20 à 30 ans après la majorité des victimes ${ }^{32}$. S'il ne sera pas question dans cet article de l'analyse de l'application de ces nouvelles dispositions légales, on pourra néanmoins garder en toile de fond, les questions inédites soulevées autour des âges et du consentement: un mineur peut-il consentir à un acte sexuel avec un majeur? Comment sortir de l'incongruité juridique de preuve du non-consentement du mineur?

\section{Cadre empirique et théorique}

13 La réalité des pratiques judiciaires à propos des violences sexuelles sur mineurs reste très peu connue, même si on assiste aujourd'hui à un développement remarquable des travaux de la sociologie du droit sur ces sujets (Mucchielli, Le Goaziou ${ }^{33}, 2009,2010$; Cromer, Grunvald, et al., 2016 $6^{34}$ ). C'est donc dans leur sillage que ma propre enquête a été réalisée afin d'éclairer la façon dont s'opèrent les changements de normes juridiques et sociales autour de la question de la qualification pénale des infractions sexuelles. Les archives judiciaires représentent un matériau extrêmement riche et précieux pour tout sociologue. Elles constituent une réserve étonnante: "toute la société s'y reflète, institutions bien sûr, mais encore économie, démographie, société, mentalités, etc. $\aleph^{35}$. L'archive judiciaire est une entrée pertinente pour notre étude socio-judiciaire des violences sexuelles sur mineurs. Ainsi, les dossiers étudiés ne prétendent pas être une représentation du système pénal français mais visent à refléter simplement une pratique du droit propre à une juridiction, dans un département donné en France au cours de l'année 2010.

14 L'enquête empirique a été menée au sein de quatre tribunaux correctionnels et pour enfants dans le sud de la France. J'ai constitué un corpus de 81 affaires, toutes jugées sur l'année 2010, pour des infractions sexuelles commises sur des mineurs. Les actes correctionnels, les plus nombreux ${ }^{36}$, concentrent de nombreuses incertitudes normatives et judiciaires : cas de correctionnalisation de viol, glissement de la frontière juridique entre l'agression sexuelle et l'atteinte sexuelle sur mineur, brouillage de la norme dans le traitement pénal de l'inceste. Les affaires relèvent aussi bien de relations entre pairs du même âge que des relations dans lesquelles l'écart d'âge est la source du scandale; de jeu ou d'initiation sexuelle entre mineurs, que des violences, parfois graves, sur des enfants et adolescents, par des mineurs ou des majeurs, dont la plupart sont issus de l'entourage proche ou de la famille. Parmi celles-ci, j'ai recensé 27 affaires intrafamiliales renvoyées devant le tribunal correctionnel et ou le tribunal pour enfants ${ }^{37}$ : une sur deux concerne des ascendants ou assimilés (beaux-pères) ${ }^{38}$, un quart des collatéraux (fratrie, cousins, oncle mineur) ${ }^{39}$, le reste des affaires impliquant des liens issus de familles recomposées (quasi-frère ou sœur, concubin de la grand-mère, père du beau-père $)^{40}$. Dans l'optique d'une lecture critique de l'application de la loi $\mathrm{n}^{\circ}$ 2010-121 du 8 février 2010, fondée sur l'usage des critères aggravants et du surqualificatif incestueux dans les infractions, j'ai choisi d'analyser qualitativement l'ensemble des affaires de violences sexuelles intrafamiliales. Seuls les dossiers entrants 
dans le cadre de l'application de la loi de 2010 ont été analysés ; celle issue de la loi du 14 mars 2016 n'a pas été prise en compte, le corpus ne s'y prêtant pas. Pour une présentation plus complète de mon corpus d'affaires, l'on peut se référer à un article paru récemment ${ }^{41}$.

15 J'ai focalisé mon travail sur l'étude du processus de qualification pénale, en l'appréhendant non pas comme indicateur de la bonne efficience du droit, mais comme "analyseur privilégié» du passage du fait au droit. L'objectif sociologique est d'analyser les enjeux de justice afin de contribuer à mettre au jour l'évolution des normes et représentations du permis et de l'interdit sexuels dans la société contemporaine. En ce sens, l'étude des dossiers judiciaires s'est avérée particulièrement riche pour mettre au jour les enjeux judiciaires en matière de traitement pénal de l'inceste. Si les peines prononcées sont évidemment importantes à la compréhension des valeurs sociales dominantes, mon hypothèse est que c'est en amont, dans ce moment de la qualification, que la sociologie peut éclairer la double dimension des affaires, entre, d'un côté, le pôle de l'appréhension des faits et, de l'autre, la preuve et le pôle des références normatives mobilisées en droit.

\section{La délicate preuve du non-consentement du mineur victime d'inceste}

16 Juridiquement, pour qu'une agression sexuelle (ou un viol) soit caractérisée, l'exigence de la preuve est double : prouver l'existence d'une situation de « violence, contrainte, menace ou surprise » et la réalisation d'un acte de nature sexuelle sur un mineur (si pénétration, ce sera un viol et dans les autres cas, une agression sexuelle). Le lien de famille et l'âge ne sont que des circonstances aggravantes. À l'inverse, pour l'atteinte sexuelle sur mineur de plus de 15 ans, infraction moins grave, le lien de famille et l'âge sont des critères constitutifs. Les règles probatoires sont beaucoup plus simples puisqu'elles n'impliquent pas de prouver l'existence d'une situation de "violence, contrainte, menace ou surprise " mais seulement la réalisation d'un acte de nature sexuelle sur un mineur. Peu importe les conditions de réalisation de l'acte, seule compte la minorité de la victime (plus de 15 ans et moins de 18 ans) et le lien avec l'auteur, constitutifs de l'infraction.

Dans les affaires impliquant des mineurs victimes d'inceste, la preuve du nonconsentement situationnel s'avère particulièrement délicate. Les magistrats sont confrontés à une difficulté majeure pour prouver l'état de contrainte ou la violence. Celle-ci est le plus souvent imperceptible, discrète, voire inaudible et la relation d'emprise empêche le plus souvent l'enfant de résister ou de s'opposer à son agresseur, quand bien même il serait en mesure de comprendre la portée des actes subis (Coste, 1997). Aussi, les deux acceptions possibles du non-consentement, le situationnel (dans tel contexte particulier) et le statutaire lié à l'âge, posent problème. Quelle qualification pénale des faits va être retenue par les juges? Celle d'agression sexuelle, la plus difficile à établir sur le plan probatoire, ou celle d'atteinte sexuelle? Les enjeux de preuve occupent donc une place centrale dans le débat judiciaire contemporain. Nous allons voir qu'ils mettent au jour un des aspects les moins visibles de ces affaires dans les recompositions normatives du permis de l'interdit sexuel: le consentement statutaire lui-même. 
De façon tout à fait étonnante, dans le corpus d'affaires de violences sexuelles intrafamiliales, le recours le plus fréquent est la qualification d'agression sexuelle sur mineurs sans qu'il y ait mention explicite des éléments situationnels (violence, contrainte, menace ou surprise). Ceux-ci sont en effet rarement présents ou mentionnés tels quels dans les jugements ou pièces judiciaires, et la contrainte morale (loi 2010) n'est jamais retenue par les juges. Comment expliquer ce phénomène ? Sur quels éléments juridiques se fondent les professionnels pour constituer l'agression sans les éléments situationnels? Pourquoi favorisent-ils l'agression plutôt que l'atteinte sexuelle plus facile à caractériser? En outre, il apparaît que l'aggravation selon la qualité de l'auteur et le qualificatif «incestueux» ne sont pas systématiquement retenus par les juges. La dimension incestueuse des affaires n'est pas toujours prise en compte par les professionnels, et certains liens familiaux (les ascendants) le sont plus que d'autres. Aussi, la pratique judiciaire donne à voir des disparités importantes dans l'appréhension pénale de l'inceste. Prenons l'exemple de plusieurs affaires pour illustrer toutes ces tensions et incertitudes juridiques.

\title{
Un beau-frère de 24 ans et sa belle-sœur de 14 ans
}

\begin{abstract}
Affaire G42. Sophie, 14 ans, déclare plusieurs mois après avoir subi des abus sexuels par Anthony, 24 ans, le petit ami de sa sœur ${ }^{42}$. Placé en garde à vue, auditionné, Anthony nie dans un premier temps puis finit par reconnaître les faits à la lecture des auditions de Sophie et des lettres qu'il lui avait envoyées. Il déclare avoir été amoureux de Sophie et ne l'avoir jamais contrainte.
\end{abstract}

19 Les faits sont établis et qualifiés dès l'ouverture de l'enquête en atteinte sexuelle sur mineur. Anthony, après avoir contesté les faits lors de sa première audition de $G^{4}{ }^{43}$, les a ensuite reconnus lors d'une $2^{\text {ème }}$ audition et devant l'expert "le sujet reconnaît avoir caressé à plusieurs reprises la sœur de sa copine, âgée de 14 ans à l'époque ${ }^{44}$. À la lecture des déclarations on apprend rapidement que suite aux graves problèmes financiers de ses parents, Sophie a été hébergée chez sa sœur Chrystelle en présence de son petit ami Anthony. Les faits s'y seraient déroulés durant plusieurs mois dans ce contexte particulier. La qualification du délit a reposé sur la référence au nonconsentement statutaire de la mineure, et cela en l'absence de contrainte, menace, violence ou surprise.

Anthony invoque un consentement mutuel devant les enquêteurs : « Il y avait un jeu de séduction entre nous [...] Elle m'a parlé d'un problème de pilosité je lui ai demandé de me le montrer elle m'a dit oui. Elle m'a montré son sexe, on était dans la salle de bain. Là je lui ai demandé juste de le toucher, au début elle a hésité à dire oui et après elle a accepté $»^{45}$. En revanche, Sophie explique s'être sentie obligée et forcée à subir les attouchements : «ça s'est produit plusieurs fois [...] Il venait me réveiller, j'essayais de faire semblant de dormir, mais il me secouait, dès fois j'ai réussi à faire semblant et là il me faisait un bisou sur le front [...] À chaque fois c'est moi qui baissais ma culotte, je me sentais vraiment obligée, mais une fois c'est lui qui l'a fait [...] Quand je voulais aller dans une autre pièce, il me rattrapait par ce qu'il pouvait, la jambe ou la main. Il n'était pas violent, il m'attrapait fort, il ne voulait pas me lâcher. En fait il n'arrêtait pas d'insister verbalement pour que je le fasse tout en me tenant pour que je ne parte 
pas $»^{46}$. L'expertise gynécologique réalisée sur Sophie, attestant de l'absence de déchirure de l'hymen, a permis d'écarter l'hypothèse du viol.

Les enquêteurs sont à la recherche d'éléments de preuve d'atteinte au consentement de Sophie lors de l'interrogatoire d'Anthony :

\begin{abstract}
- Question du brigadier : Comment expliquez-vous ce qui vous a conduit à avoir une relation amoureuse avec Sophie? Anthony: J'étais mal dans ma relation de couple, elle ressemblait beaucoup à sa sœur. Sophie m'aguichait, j'ai cédé, j'ai craqué ${ }^{47}$.

- Question de l'enquêteur: Sophie a-t-elle pleuré ou avez-vous senti à un moment qu'elle n'était pas en accord avec les caresses que vous lui faisiez? Anthony: Lorsque je la caressais son sexe était mouillé, j'avais donc la sensation que Sophie ressentait du plaisir. Je n'ai pas souvenir qu'Sophie ait pleuré... Question de l'enquêteur: Avez-vous contraint Sophie d'une manière ou d'une autre? Anthony: Non, je n'ai jamais contraint Sophie, elle venait vers moi sans peur. Je n'ai jamais usé de violences, de menaces. D'ailleurs j'ai arrêté de caresser Sophie lorsque j'ai vu qu'elle était gênée. J'ai cessé immédiatement et je n'ai plus jamais recommencé. J'ai d'ailleurs pleuré ce jour-làa ${ }^{48}$.
\end{abstract}

22 À la lecture du dossier, on apprend qu'Anthony était amoureux de Sophie, deux lettres écrites de sa main sont jointes au dossier, en voici quelques extraits : "J'aime vraiment la relation qu'on a tous les deux; tu peux même pas t'imaginer comme tes attentions (massages, câlins et caressages) me font plaisir, des fois, j'ai même un peu honte de t'en demander mais c'est tellement agréable que je te demande quand même. Je pense que tu devrais pas t'en faire autant... sur ces bonnes paroles je te laisse en attendant avec impatience la prochaine attention $»^{49}$. Pour l'expert psychologue qui a examiné Sophie, il est évident que ce contexte amoureux a été très perturbant pour la jeune fille « elle semble déstabilisée par cette relation qui au départ était une relation amicale [...] elle s'est sentie piégée par ce garçon $»^{50}$.

Le parquet a décidé du renvoi direct devant le TC pour atteinte sexuelle sur mineur de 15 ans pour « avoir à ... entre le 3 septembre 2008 et le 17 mars 2009 .... Exercé sans violence, ni contrainte, ni menace ni surprise, une atteinte sexuelle sur la personne de Sophie ... mineure de moins de 15 ans comme étant née le ../../1994 en l'espèce en caressant à plusieurs reprises le sexe $»^{51}$. Anthony a été placé sous contrôle judiciaire jusqu'à sa comparution devant le TC. Lors du procès, le ministère public a requis 2 ans d'emprisonnement avec sursis partiel à hauteur de 6 à 9 mois et l'avocat d'Anthony a plaidé l'indulgence. La juridiction de fond a suivi les réquisitions du parquet et condamné Anthony à 18 mois d'emprisonnement dont 12 avec sursis assorti d'une mise à l'épreuve pendant 3 ans, avec l'obligation de se soumettre à des soins. Le tribunal a également prononcé à titre complémentaire l'inscription au FIJAIS ${ }^{52}$.

Dans cette affaire, la qualification pénale ne change pas tout au long de la procédure. Les actes ont été qualifiés d'atteinte sexuelle sur mineur de 15 ans. Il n'y a eu ni aggravation par personne ayant autorité, ni d'ajout du sur-qualificatif « incestueux » à l'infraction. Si la dimension incestueuse de l'affaire n'a pas du tout été prise en compte, c'est que l'inceste de $2^{\text {ème }}$ type ${ }^{53}$ a été considéré comme étant moins grave que d'autres. Nous formulons l'hypothèse que différents éléments issus du dossier ont conduit à faire pencher les professionnels en faveur de la qualification pénale d'atteinte sexuelle plutôt que d'agression: les types de liens entre auteur et victime, la proche majorité sexuelle de Sophie, le contexte de séduction, le relatif jeune âge adulte d'Anthony et 
son profil qui n'est pas du tout celui d'un délinquant sexuel. On est dans l'idée d'une d'atténuation de l'acte transgressif, et la logique qui prévaut est exclusivement celle du non-consentement statutaire lié l'âge du mineur. Ce qui prime c'est l'interdit des âges majeur/mineur, comme le rapporte Anthony lors de son procès «J'ai compris que c'était une faute grave. J'aurais dû stopper aussitôt cette relation parce qu'elle est mineure $»^{54}$.

Néanmoins, dans la majorité de notre corpus d'affaires de violences sexuelles intrafamiliales, le recours à l'agression sexuelle est massif et ce même en l'absence de précisions juridiques sur le contexte de violence ou de contrainte exercé sur l'enfant. Or, il n'existe pas dans le droit pénal français de présomption légale de nonconsentement du mineur victime qui conduirait de facto à la qualification d'agression sexuelle. Le législateur n'a en effet pas fixé de seuil d'âge en dessous duquel il y a une présomption irréfragable de non-consentement, à la différence d'autres pays européen $\mathrm{s}^{55}$; et le lien de famille n'est pas constitutif de l'agression sexuelle. En France, ce n'est que depuis 2005 que la Cour de cassation a estimé que le très jeune âge des enfants, jusqu'à 6 ans ${ }^{56}$, pouvait constituer une contrainte: le manque de discernement dû au très jeune âge de l'enfant « les rendait incapables de réaliser la nature et la gravité des actes qui leur sont imposés $\aleph^{57}$. La jurisprudence considère que «du fait de leur âge, les victimes avaient été dans l'impossibilité de manifester la moindre adhésion à des comportements dont elles n'avaient pu saisir la portée $»^{58}$.

Dans nos dossiers ${ }^{59}$, on retrouve cette présomption irréfragable d'un non-consentement fondé sur le très jeune âge du mineur victime d'inceste. Mais la pratique judiciaire tend à montrer que différents âges sont concernés ( 7 ans, 12 ans, 14 ans, 15 ans et demi) selon les liens de famille avec l'auteur et selon les écarts d'âge en particulier entre mineurs. La question des âges du mineur victime acquiert une place inédite dans la qualification pénale sans que soit caractérisé explicitement la "violence, contrainte, menace ou surprise ». Le critère de l'âge, mais aussi celui d'ascendance ou d'autorité, sont retenus à la fois comme élément constitutif et aggravant de la même infraction d'agression sexuelle. Voyons les affaires.

\title{
Un père et sa fille de 7 ans
}

\begin{abstract}
Affaire N20. Emilie, 13 ans, confie à son professeur avoir été abusé sexuellement par son père, Patrick, lorsqu'elle avait 7 ans. Elle a déclaré aux enquêteurs que son père avait l'habitude de venir dans sa chambre, de lui caresser les seins et les fesses et de s'allonger sur elle. Les faits sont niés par son père.
\end{abstract}

Dans le procès-verbal de transmission du parquet destiné au commissariat de police, l'on trouve une qualification d'agression «Procéder à une enquête sur les faits de viols et/ou agressions sexuelles dénoncés dans le signalement joint concernant la jeune Emilie $^{60}$ ». À l'ouverture d'enquête, la qualification initiale est celle d'atteinte sexuelle sur mineur de 15 ans par ascendant, et non d'agression. Au cours de l'audition d'Emilie, les enquêteurs veulent s'assurer de ne pas passer à côté d'un possible viol :

Question de l'enquêteur : Est-ce que tu sais ce que c'est que le viol ?

Emilie : Pas trop, je n'ai pas tellement appris ça.

L'enquêteur: Le viol, c'est toute pénétration que l'on peut faire subir à une personne non consentante, mineure ou majeur, par le sexe ou par quelque 
chose d'assimilé au sexe. Je m'explique : un sexe d'homme qui pénètre le sexe d'une femme, le sexe d'un homme qui pénètre les fesses d'une femme (l'orifice) ou un objet qui pénètre le sexe ou les fesses, par exemple une bouteille, ou même un doigt qui pénètre le sexe ou les fesses, et même un sexe qui pénètre la bouche peut être considéré par la loi comme un viol. Astu bien compris?

Emilie : Oui

L'enquêteur : Penses-tu avoir été victime enfant ou même plus récemment, de ce type de fait de ton père, notamment quand tu as dormi avec lui ?

Emilie : Non

L'enquêteur : Te rappelles tu comment tu étais vêtue pour dormir?

Emilie : D'un pyjama avec des nounours

L'enquêteur : Tu étais habillée en te levant le matin?

Emilie : Oui

L'enquêteur à Emilie : Te rappelles tu avoir eu mal aux fesses ou au sexe en te réveillant le matin?

Emilie : Non ${ }^{61}$.

L'hypothèse du viol est écartée par les enquêteurs. Le professeur ayant reçu les confidences de l'enfant est auditionné et décrit un contexte de soumission de l'enfant: «Je lui ai demandé si un jour elle avait dit non, elle a répondu que non. Elle avait dit qu'elle avait essayé mais que finalement elle se résignait elle ne pouvait pas lutter parce que c'était son père, et qu'elle l'aimait, elle ne pouvait pas lutter et que c'était comme ça [...] $\rrbracket^{62}$. Les enquêteurs auditionnent également l'ami d'Emilie qui a été son premier confident sur le contexte des faits et leur révélation : "Question de l'enquêteur à Florian: Est-ce que ce qu'elle t'a dit t'es apparu comme une agression sexuelle? Réponse de Florian : Il me semble qu'il ne l'a pas touché mais qu'il a essayé mais je ne suis pas vraiment sûr. Question de l'enquêteur à Florian : T'a-t-elle reparlé de ça après septembre-octobre ? Réponse de Florian : Non. Question de l'enquêteur à Florian : T'a-telle dit ce qu'elle pensait de tout ça ? Réponse de Florian: Non ${ }^{63}$. Quant à l'expertise psychologique d'Emilie, celle-ci confirme l'existence de «trace patente d'effraction psychique en rapport avec une force traumatique d'ordre sexuel ».

Le père nie massivement les faits d'attouchements sexuels lors de sa garde-à-vue, puis admet, lors de sa dernière audition, s'être rendu dans la chambre de sa fille : «Si Emilie dit qu'elle a subi des attouchements c'est peut-être vrai, mais je n'arrive pas à m'en rappeler, si elle me le dit d'elle-même je l'admettrai " ${ }^{64}$. L'expertise psychiatrique révèle un tableau d'alcoolisme chronique tout en précisant que «le sujet nie les faits $»^{65}$.

En fin d'enquête préliminaire, la qualification change en agression sexuelle sur mineur de 15 ans par ascendant. Le parquet décide de l'ouverture d'une information judiciaire, et saisit un juge d'instruction. Rien n'est noté dans le dossier sur le renoncement à l'atteinte sexuelle sur mineur. On peut supposer ici que le contexte incestueux de l'affaire et les dénégations massives du père ont été déterminants.

En effet, les aveux du père sont laborieux. Lors de sa première comparution devant le juge d'instruction, il concède avoir eu une pulsion tout en affirmant ne jamais être allé au-delà. Il se rétracte ensuite massivement dans un courrier adressé au juge : «Je ne suis jamais allé dans la chambre de ma fille Emilie pour lui retirer son bas de pyjama. Je n'ai jamais pratiqué sur elle des caresses à caractère sexuel [...] $»^{66}$ et se maintient dans ses dénégations lors de la confrontation avec sa fille. Il persiste devant le nouvel expert psychiatre "parce que je ne l'ai pas fait. Moi c'est certain ${ }^{67}$. Il conteste même la 
qualification pénale « une agression sexuelle, ça voudrait dire qu'elle a des bleus sur les bras! $\aleph^{68}$. Différentes auditions sont réalisées sur commission rogatoire, la mère d'Emilie, son frère, sa tante, de nouveau Emilie, son petit ami : tous corroborant les déclarations d'Emilie sur le contexte des faits et leur révélation. Les nouvelles expertises confirment l'absence de toute affabulation et attestent d'un traumatisme sexuel chez Emilie.

Le juge décide de renvoyer le père devant le TC pour agression sexuelle incestueuse sur mineur de 15 ans par ascendant, mais sans rien expliciter sur l'existence d'une "violence, contrainte, menace ou surprise ». En revanche, il a mentionné dans son $\mathrm{ORTC}^{69}$ le contexte incestueux de l'affaire et l'alcoolisation du père : «Patrick s'est livré dans ses déclarations à des expressions pour les moins troublantes, venant étayer les conclusions de l'expert psychiatre selon lesquelles le mis en examen perçoit le petit ami de sa fille comme un rival, ce qui tendrait à démontrer des tendances incestueuses dont la mise à exécution a été facilitée par le contexte d'alcoolisme, notamment lorsque le mis en examen nous indiquait «Emilie était avec moi car elle n'avait pas de petit ami à l'époque [...] l'expert relève un climat incestuel transparaissant notamment à travers les remarques faites par celui-ci à l'égard des petits amis de sa fille, lesquelles s'inscrivent manifestement dans un contexte de concurrence et de jalousie. Ce comportement incestueux étant favorisé par le contexte d'alcoolisme $»^{70}$. Le père a été condamné à 4 ans d'emprisonnement dont 30 mois avec sursis et à titre complémentaire à une inscription au FIJAIS. Emilie a obtenu 25000 euros de dommages et intérêts et le remboursement de 6240 euros de frais médicaux.

Le renoncement à la qualification d'atteinte sexuelle au profit de l'agression sexuelle n'est pas explicité. En revanche, il a bien été relevé l'état de minorité de la victime, le contexte incestueux des faits, le profil du mis en cause et ses dénégations massives. Face à l'horreur de l'inceste intergénérationnel, prévaut une présomption irréfragable de non-consentement. Mais sans caractériser en quoi il y a eu un non-consentement situationnel, les critères d'âge et d'ascendance, sont retenus à la fois pour aggraver et constituer la même infraction. Ce phénomène des qualifications enchevêtrées est récurrent dans nos dossiers. Notre hypothèse est que tout acte de nature sexuelle perpétré par un ascendant sur un mineur est considéré comme plus "grave » et conduit de facto à la qualification d'agression sexuelle. Dans l'affaire suivante, la même logique s'engage. Des actes commis sans que la contrainte soit prouvée, par un homme sur son fils de 12 ans, sont qualifiés d'agression sexuelle.

\title{
Un homme et son fils de 12 ans, ainsi que son neveu de 14 ans
}

\author{
Dans l'affaire N5, Yannick, 12 ans, révèle 15 jours après les faits à sa mère, \\ avoir subi des abus sexuels par son père, Didier. Les parents sont séparés. \\ L'enquête met au jour une deuxième victime, Jean-Pierre, le neveu de Didier, \\ qui aurait subi lui aussi des abus sexuels quelques années auparavant, alors \\ qu'il était âgé de 14 ans. Le père est condamné à 3 ans \\ d'emprisonnement avec sursis et suivi socio-judiciaire pendant 5 ans, une \\ inscription FIJAIS et un retrait total de l'autorité parentale sur Yannick.
}

Le père conteste formellement les faits. À la $1^{\text {ère }}$ audition, il nie totalement : « pour moi je n'ai rien fait (...) c'est-à-dire que moi je ne pense pas avoir fait des trucs à mon fils de 12 ans ", puis en plein milieu d'audition, il reconnait les faits commis sur Yannick, son fils (première victime) tout en précisant qu'il ne l'a fait que sur lui et pas sur ses deux 
autres fils, expliquant qu'ils étaient trop jeunes «peut-être que je l'aurais fait s'ils avaient le même âge ». À la $2^{\text {ème }}$ audition, Didier précise que depuis son divorce il est attiré par les hommes, il évoque son homosexualité à l'enquêteur, tout en précisant ne jamais avoir eu de relations sexuelles avec un homme. Il avoue spontanément les faits commis sur Jean-Pierre, son neveu (la deuxième victime). Enfin, à la $3^{\text {ème }}$ audition, Didier précise qu'il consulte régulièrement un site internet homosexuel, les résultats de l'expertise informatique de son ordinateur venant de lui être révélés : " présence dans les archives du navigateur web de vidéos pornographiques de type homosexuel (...) multitudes d'images pornographiques à caractère homosexuel ».

L'enquête est menée rapidement, en un mois, pas d'instruction et l'affaire est renvoyée en jugement le mois suivant sur comparution du parquet. Une seule expertise psychologique est réalisée, sur Yannick; elle relève que ce dernier a une "méconnaissance totale du registre sexuel; ce qui est inhabituel pour un préadolescent mais qui pourrait traduire sa gêne concernant ce sujet et la charge émotionnelle anxiogène quant aux faits allégués (...) un suivi psychologique serait opportun ». L'absence de connaissance sexuelle va s'avérer déterminante pour fonder le non-consentement, bien que le mineur soit âgé de 12 ans. La contrainte pouvant résulter, rappelons-le selon la jurisprudence, du très jeune âge de l'enfant, soit 6 ans. S'agissant de la deuxième victime, le neveu Jean-Pierre, 14 ans au moment des faits, rien n'est mentionné au dossier. On suppose ici, que l'oncle ayant reconnu les faits, le défaut de consentement se trouve fondé par les critères d'âge et de qualité d'auteur, comme dans l'affaire précédente.

Ainsi, l'auteur est condamné pour agression sexuelle incestueuse sur mineur de $\mathbf{1 5}$ ans par ascendant pour les faits commis sur son fils et par personne ayant autorité pour les faits commis sur son neveu. L'extrait de jugement rappelle les faits et succinctement la règle juridique pour fonder l'agression sexuelle : «Didier, prévenu d'avoir à ... les 25, 26 et 27 septembre 2009 ainsi que les 9, 10 et 11 octobre 2009, par violence, menace, contrainte ou surprise, commis ou tenté de commettre des agressions sexuelles sur la personne de Yannick (...) en l'espèce en le masturbant avec ces circonstances que la victime était mineure de 15 ans pour être née le ..././1997 et que les faits ont été commis par ascendant [...] D'avoir à ... dans le premier semestre 2003, par violence, menace, contrainte ou surprise, commis ou tenté de commettre des agressions sexuelles sur la personne de Jean-Pierre (...) en l'espèce en lui touchant le sexe avec ces circonstances que la victime était mineure de 15 ans pour être née le ../../ 1988 et que les faits ont été commis par une personne ayant autorité. Faits prévus et réprimés par les articles 222-30, 29-1, 222-22 ». Là encore, on peut en conclure que les critères de l'âge, lié ici à la vulnérabilité de Yannick (méconnaissance du registre sexuel), et de qualité d'auteur, s'avèrent déterminants pour retenir l'infraction. L'ajout du surqualificatif incestueux et de l'aggravation viennent souligner distinctement la dimension de l'inceste.

\section{Un grand-père et sa petite fille de 15 ans et demi}

\footnotetext{
Affaire N15 : Léa 15 ans et demi révèle immédiatement à sa tante des attouchements sexuels qu'elle vient de subir de la part de son grand-père paternel, Jacques 78 ans. La tante accompagne sa nièce aux urgences pédiatriques, l'affaire est aussitôt signalée au Parquet.
} 
L'enquête a établi la matérialité des faits: caresses sur la poitrine et le sexe, cunnilingus. Léa, 15 ans et demi, est majeure sexuellement au moment des faits ce qui exclut la référence à un non-consentement statutaire seulement lié à l'âge. Tout l'enjeu pour les professionnels qui auditionnent Léa, est de caractériser en quoi il y a eu contrainte :

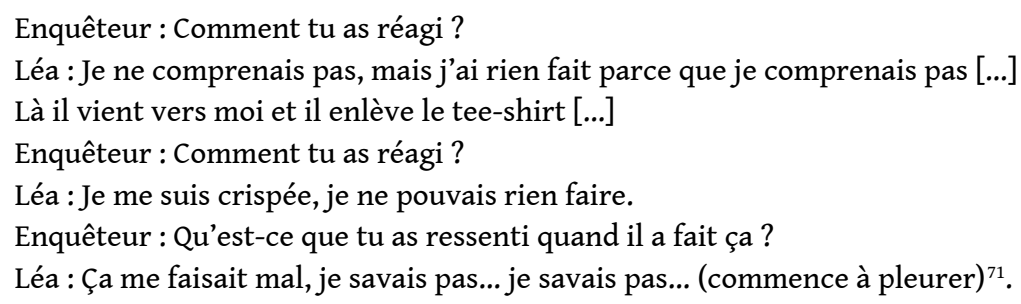

La thèse d'une sidération psychique est avancée par l'expert psychologue pour expliquer l'absence de réaction de Léa: "Elle évoque sa peur avec un effet de sidération. Elle ne savait pas quoi faire, laissant transparaître un sentiment de culpabilité $»^{72}$. Elle est étayée par le profil psychologique fragile de Léa : «présence d'une symptomatologie dépressive ancienne réactivée par les agressions sexuelles subies $»^{73}$. En outre, Léa ayant indiqué lors de son audition ne jamais avoir eu de rapports sexuels, une expertise gynécologique est réalisée et atteste l'absence d'hymen déchiré. L'hypothèse du viol est écartée.

Les faits sont rapidement reconnus par le grand-père au cours de sa GAV, mais il conteste toute contrainte «Je lui ai dit on va passer à la maison [...] C'est là que ça s'est passé, à la maison elle s'est déshabillée [...] ça m'a pris comme ça, je ne peux pas l'expliquer. Je n'avais pas fermé à clef la porte $\aleph^{74}$. Jacques évoque devant l'expert une séduction de la part de Léa, voire une provocation de cette dernière. L'expert assimile cette posture à des traits de perversion : «On peut décrire chez lui des comportements pervers, qui l'ont amené à imposer à sa petite fille des attouchements sexuels qu'il reconnait tout en les rationalisant partiellement [...] Le discours de Jacques suggère de sa part la recherche d'une atténuation de sa propre responsabilité en "chargeant" l'adolescente [...] On peut donc parler de pédophilie à caractère incestueux ${ }^{75}$.

En outre, on apprend que le grand-père aurait commis dans le passé des viols sur deux tantes paternelles de Léa : «J'avais banni tout cela de ma mémoire, je ne sais même pas quand il a arrêté de me violer. Je me souviens que quand il me faisait subir ses viols il me disait qu'il faisait mon éducation $»^{76}$. Les enquêteurs l'ont précisé dans leur rapport de synthèse: "Il était également établi qu'il a violé ses deux belles-filles dans leur enfance mais les faits sont prescrits à ce jour $»^{77}$. Le père de Léa, auditionné après la déposition de sa fille, déclare que son père était violent durant son enfance, et sa mère alcoolique.

41 La procédure est expéditive. Au terme de deux jours d'enquête, le parquet décide du renvoi de l'affaire devant le TC pour agression sexuelle par ascendant pour « avoir à... [...] le 28/07/2008, commis une atteinte sexuelle avec violence, contrainte, menace ou surprise sur la personne de Léa, âgée de 15 ans comme née le ... 1993 en procédant sur elle à des attouchements de nature sexuelle (attouchements de sa poitrine de son sexe, cunnilingus...) avec cette circonstance que les faits ont été commis par ascendant (grand-père paternel) $\aleph^{78}$. L'agression est établie, mais rien ne vient expliciter en quoi il 
$\mathrm{y}$ a eu contrainte. Le grand-père persiste à contester la contrainte, y compris au cours de son procès :

La présidente du Tribunal : "Je sais que tu en as envie" cela sort d'où ? Vous croyez qu'une fille de 15 ans a envie d'un vieux monsieur?

Le prévenu : je ne sais pas. Ce n'est pas de ma faute, je ne sais pas ce qui m'a pris. Oui j'ai des regrets? J'avoue ce que j'ai fait ${ }^{79}$.

Il a été condamné à 3 ans d'emprisonnement dont 24 mois de sursis avec interdiction de rencontrer la victime, d'exercer une activité même bénévole impliquant un contact habituel avec un mineur, avec suivi socio-judiciaire pendant 5 ans, inscription FIJAIS et privation de tous les droits civiques, civils et de famille pendant 5 ans. Parallèlement 65000 euros $^{80}$, au titre de dommages et intérêts, ont été octroyés à Léa ${ }^{81}$ pour réparation du préjudice moral et « corporel ».

L'intérêt de cette affaire tient au choix de la qualification pénale d'agression sexuelle plutôt que d'atteinte. En effet, il n'a pas été retenu celle d'atteinte sexuelle sur mineur de plus de 15 ans par ascendant, alors qu'elle est plus facile à caractériser, et de surcroit la seule à réprimer spécifiquement l'inceste. Comme cela s'est vérifié dans d'autres affaires, on est dans l'horreur de l'inceste intergénérationnel. Ainsi l'acte est considéré comme étant plus grave ${ }^{82}$ y compris si la victime mineure est âgée de plus de 15 ans. Les professionnels ont considéré ici que la relation incestueuse du grand-père avec sa petite fille de 15 ans et demi est nécessairement une violence et donc une agression sexuelle. Sur le plan juridique, les critères d'âge et de lien de famille sont pris en compte à la fois pour aggraver et constituer la même infraction d'agression sexuelle. Ce faisant, coexistent ensemble les deux formes de non-consentement, que le législateur avait voulu séparer: le statutaire lié à l'âge et le situationnel lié aux conditions de l'infraction.

\section{Un demi-frère de 15 ans et sa demi-sœur de 13 ans (entre mineurs)}

4 Il en est tout autrement, dans le cas des violences sexuelles perpétrées par des mineurs sur d'autres mineurs : le droit pénal n'a rien prévu pour les atteintes sexuelles qui ne se doublent pas d'agression ${ }^{83}$. En outre, à la différence d'autres législations européennes ${ }^{84}$, le droit ne tient pas compte des écarts d'âge entre mineurs pour fonder la contrainte. Aussi, la preuve du non-consentement du mineur s'avère encore plus délicate. Dans la pratique judiciaire, à l'échelle du corpus étudié, le très jeune âge de la victime et les écarts d'âge entre les mineurs, auteurs et victimes, sont retenus pour fonder la contrainte. Mais lorsque les âges sont proches, la présomption de non-consentement est moins évidente. C'est particulièrement significatif entre un demi-frère et une demisœur d'âge proche dans une famille recomposée. Voyons l'affaire.

\footnotetext{
Affaire G26. Sabine, âgée de 13 ans, révèle à sa mère avoir été violée par son demi-frère, Jordan, âgé de 15 ans, sur une plage de la commune, alors qu'elle se trouvait en vacances en juillet $2005 \mathrm{chez}$ leur père. De même, elle déclare avoir subi le jeu sexuel de la taupe lorsqu'ils étaient enfants ${ }^{85}$. Jordan conteste formellement les faits et invoque des attouchements sexuels réciproques et consentis.
}

Sabine et Jordan sont mineurs, ils sont frère et sœur consanguins ${ }^{86}$, de peu d'écart d'âge et pubères. Cet aspect est en effet un des points soulevés dans l'expertise 
gynécologique et dans les auditions (père, mère et belle-mère). Sabine est décrite comme ayant eu un développement précoce et paraissant plus âgée que son âge. À l'ouverture d'enquête, la qualification de viol sur mineur de $\mathbf{1 5}$ ans est retenue, suite aux déclarations de la mère de Sabine : «Je me présente à votre service afin de déposer plainte contre le fils de mon ex-compagnon, prénommé Jordan âgé de 16 ans et demeurant ... pour le viol de ma fille Sabine âgée de 14 ans [...] Elle m'a dit qu'elle avait été à la plage en juillet dernier avec son demi-frère. Il l'a pénétré avec son sexe et il lui disait de le laisser terminer ${ }^{87}$. Le médecin légiste qui examine Sabine accrédite la thèse d'un viol: «en conclusion, l'examen médical de Sabine a mis en évidence une déchirure hyménée ancienne sur un hymen complaisant. Ces aspect peut être en rapport avec une tentative de pénétration sexuelle $»^{88}$.

Quant à Jordan, il reconnaît les attouchements sexuels avec sa sœur mais sur la base d'un consentement réciproque, et conteste formellement toute pénétration. Pour le père des adolescents, il n'y a pas eu viol, mais attouchements consentis : « Nous avons ensuite confronté Jordan et Sabine, ils ont reconnus les attouchements réciproques et consentis... Sabine a redit devant le spécialiste qu'il n'y avait pas eu pénétration. La psychologue en a conclu qu'il s'agissait d'une sorte "d'attouchements découverte " La belle-mère de Sabine et mère de Jordan : "Comment expliquez-vous les accusations de Sabine? Réponse: Déjà pour moi il n'y a jamais eu viol, mais attouchements réciproques et consentis. Question: Savez-vous que Sabine n'avait que 13 ans à cette époque et que son consentement ne vaut rien? Réponse : Mon fils n'avait que 15 ans et il faut voir Sabine, elle est aussi grande que Jordan et bien charpentée $»^{90}$.

$\mathrm{Au}$ cœur de cette affaire se trouve le problème de la preuve matérielle du viol. Étonnamment, le fond de la problématique incestueuse n'est pas du tout évoqué. $\mathrm{Ni}$ dans l'expertise psychiatrique de Jordan, centrée sur l'absence de dangerosité, ou psychologique: «Il ne peut expliquer les raisons pour lesquels il n'a pas repoussé sa sœur, ni pourquoi il a glissé sa main sous son maillot [...] Ultérieurement durant l'année 2005 il serait retourné à la plage avec sa demi-sœur sans aucun incident $»^{91}$. Aucune expertise psychologique de Sabine n'a pu être réalisée, celle-ci ne s'étant pas présentée aux rendez-vous. Au terme de la première enquête, les faits sont qualifiés de viol sur mineur de 15 ans et l'affaire est ainsi résumée : « La victime déclare que se trouvant en vacances en juillet 2005 chez son père à ..., son demi-frère l'a violé sur une plage de la commune, en la pénétrant au moins une fois avant de finir de se masturber devant elle. Le mis en cause nie les faits, et reconnait qu'il y a eu attouchements sexuels mais réciproques et consentis. Il est déféré au parquet de ... sur instruction du procureur en vue de sa mise en examen $»^{92}$. Le parquet décide de l'ouverture d'une information judiciaire et retient deux chefs d'inculpation contre Jordan : le viol sur mineur de $\mathbf{1 5}$ ans pour les faits allégués les plus récents et l'agression sexuelle sur mineur de $\mathbf{1 5}$ ans pour le jeu de la taupe ${ }^{93}$. Jordan est mis en examen et placé sous contrôle judiciaire. La perte de virginité de Sabine fait l'objet d'interrogations. Devant le magistrat instructeur, Jordan confirme de nouveau le consentement mutuel : « Tout d'abord il n'y a pas eu viol. Il y a eu des attouchements sexuels consentants dans les deux cas. Ce que je veux dire c'est que nous étions elle et moi consentants $»^{94}$. Le juge d'instruction émet une supposition sur la perte de virginité de Sabine : « Le juge : Sabine a fait l'objet d'un examen gynécologique qui met en évidence "une déchirure ancienne sur un hymen complaisant". Faut-il comprendre que Sabine met à profit une relation sexuelle qu'elle 
aurait eu avec quelqu'un d'autre que vous pour vous imputer un viol que vous n'avez pas commis ? Jordan : oui $»^{95}$.

Une confrontation est organisée entre Sabine et Jordan. Jordan reconnaît des caresses mutuelles et réciproques, y compris lors du jeu du docteur. Sabine évoque désormais une pénétration minimale "un petit peu $»^{96}$ sans rapport sexuel complet. Une hypothèse est avancée par les enquêteurs, celle d'un possible contexte amoureux. On retrouve ces éléments dans différentes auditions. Celles d'une cousine: «Lorsque Sabine te parlait de son demi-frère te disait-elle si elle le trouvait beau ou si elle était amoureuse de lui ? Réponse: Non pas du tout. Question : À ton avis est-il possible que Sabine ait eu un rapport sexuel consenti avec Jordan et que par la suite elle dit avoir été violée par peur des conséquences? Réponse : Je ne pense pas... c'est vrai que j'ai vu Jordan et Sabine pendant la foire de... des vacances de pâques $2006[\ldots]{ }^{97}$.

Dans cette délicate affaire d'inceste s'entremêlent deux problèmes : la perte de la virginité de Sabine sans savoir si elle incombe au frère, et la question du consentement de Sabine. Au terme des investigations, le juge ne retient pas le viol et requalifie les faits en agressions sexuelles sur mineur de $\mathbf{1 5}$ ans "Dispositions aux fins de requalification: attendu que les faits qualifiés de viol, survenus à ... en juillet 2005, s'analysent plus exactement en agressions sexuelles. Disons requalifier en ce sens. Dispositions aux fins de renvoi devant le tribunal pour enfant: Attendu qu'il résulte de l'information des charges suffisantes contre Jordan d'avoir à ... courant juillet 2005... par contraintes, menaces, surprises ou violences, commis une atteinte sexuelle en l'espèce en glissant sa main dans le maillot de bain de la victime et en lui pratiquant des caresses sur le sexe puis en se frottant contre son sexe, au préjudice de Sabine mineure de moins de 15 ans comme étant née le .. /../1992 $»^{98}$. C'est un cas de correctionnalisation de viol. Jordan est renvoyé devant le TPE et non une cour d'Assises pour mineurs. Or, il n'a pas été condamné, les juges de fond ayant prononcé la relaxe.

51 Cette affaire illustre le problème de la preuve. Jordan reconnait les attouchements mais pas le viol. Sabine déclare avoir été violée et avoir subi un rapport sexuel complet. L'examen gynécologique atteste que la jeune fille a « un hymen de type complaisant et une déchirure profonde». Cet examen, qui semble corroborer la version de la jeune fille, entraine la conviction du juge. Puis les mineurs sont confrontés, la jeune fille revient sur ses propos, ne parle plus de pénétration complète ni de douleur et il y a une discussion sur la perte de virginité de Sabine. Le doute sur ses premières allégations s'installe et une deuxième hypothèse succède à la première: on ne peut pas comprendre cette affaire sans préciser que la jeune fille est d'origine magrébine par sa mère. Elle sait que dans sa famille la perte de virginité avant le mariage n'est en aucun cas acceptée, comme le précise sa mère dans un courrier qu'elle adresse au Procureur « ma fille a perdu à 13 ans ce que l'on a de plus précieux. L'innocence et le respect de soi-même [...] elle subira une opération afin de reconstruire son hymen ${ }^{99}$. Pour Sabine aussi, il est difficile d'avouer à sa mère sa perte de virginité et elle peut vouloir faire porter à son demi-frère la perte de sa virginité. Dans cette hypothèse, après le changement de ses déclarations, le soupçon pèse sur elle et les juges ont pu avoir en tête ce schéma culturel : une fille maghrébine doit être vierge et si elle ne l'est plus, il n'est pas impossible qu'elle se saisisse d'un prétexte pour se disculper ou pour faire porter la responsabilité à un "violeur ». On soupçonne donc qu'elle accuse son demifrère pour se disculper, avant de revenir sur ses déclarations en disant non puis un « petit peu». Le juge ne retient plus la pénétration vaginale ni sa preuve (il est dit 
désormais que "l'examen gynécologique ne peut être retenu à distance des faits ») et ne prend en compte qu'une partie des faits (caresses sur les seins). Il requalifie selon la version de Jordan. En outre, la dimension de l'inceste est connue, mais entre demi-frère et demi-sœur, les juges ne cherchent pas à le sanctionner. Si Jordan avait été le beaupère ou le père cela aurait été différent. Au final Jordan a été relaxé par le TPE, bien qu'il ait reconnu des attouchements sexuels sur sa sœur, dans l'enfance et à l'adolescence. L'inceste consenti entre mineurs n'a pas été puni. Celui commis au sein de la fratrie parait moins grave. Ainsi, on sanctionne les relations entre ascendants et descendants comme on l'a vu dans les affaires précédentes, mais on n'a pas le même regard sur l'inceste entre demi-frères et sœurs, ni entre beau-frère et belle-sœur, comme dans la première affaire présentée dans cet article.

\section{Conclusion}

52 À la lumière de ces différentes affaires, on perçoit toute la complexité de la notion de non-consentement du mineur victime d'inceste. Si la tendance en Europe est à l'affirmation que la perpétration d'abus sexuel sur un mineur par un membre de la famille ou une personne ayant autorité est un élément constitutif de l'infraction et non une circonstance aggravante, la pratique judiciaire de ces deux tribunaux tend à s'en rapprocher.

Nos résultats montrent en effet que les professionnels ont rarement recours à la qualification d'atteinte sexuelle alors qu'elle est la plus facile à caractériser en matière probatoire (absence de violence, contrainte, menace ou surprise). En revanche, ils retiennent massivement l'agression sexuelle, mais sans que l'on sache explicitement, dans les pièces judiciaires (réquisition, jugement), en quoi les faits ont été commis avec "violence, contrainte, menace ou surprise ». Ce faisant, les critères d'âge (minorité de 15 ans) et de lien d'ascendant deviennent à la fois des éléments aggravants et constitutifs de la même infraction. Ce phénomène juridique, bien connu des juristes ${ }^{100}$, les enchevêtrements des qualifications, est un trait commun à nos dossiers. Il se substitue complètement à l'usage de la contrainte morale, telle que prévue par le législateur (loi 2010), et qui a fait l'objet de vives critiques par la doctrine jusqu'à la récente décision du Conseil constitutionnel du 6 février $2015^{101}$.

En somme, les professionnels fondent les agressions sexuelles à partir des deux formes de non-consentement: le statutaire lié à l'âge et le situationnel lié aux circonstances de l'infraction. En nous référant à la figure de la hiérarchie, telle que développée par le sociologue Louis Dumont, dans son ouvrage Homo Hierarchicus, nous dirions que selon la situation, une dimension englobe l'autre sans pour autant la nier. Dans notre cas, l'agression englobe l'atteinte sexuelle. N'est-ce pas contraire à la logique d'opposition entre les infractions qui a été voulue par le législateur dans le nouveau code pénal de 1994 (cf. infra) ? La pratique judiciaire vient donc bousculer les catégories du droit; la frontière juridique entre l'agression et l'atteinte sexuelle apparaît véritablement de plus en plus floue. Tous ces changements ne sont pas sans conséquence pour la définition même de la prohibition pénale de l'inceste, qui se trouve de plus en plus englobée dans un interdit des âges. Dans ces considérations nouvelles, la question de l'âge acquiert une place inédite. On voit se profiler différents statuts d'âges, avec plus ou moins de force de présomption de non-consentement du mineur victime selon la configuration relationnelle (lien victime/auteur). La 
présomption de non-consentement du mineur victime d'inceste apparaît de plus en plus liée à une indicible frontière : la barrière sacralisée des âges. Elle substitue celle des places et redéfinit tout autrement les rapports d'âge et de générations. Ces changements reflètent à la fois une construction idéologique de notre société qui tend à protéger davantage les personnes les plus vulnérables que sont les mineurs mais aussi à réaffirmer un interdit majeur : l'asymétrie des âges mineur/majeur.

Les vifs débats et controverses ayant entouré l'article 2 du projet de loi sur les violences sexuelles et sexistes en 2018 en France, sur la possibilité d'une présomption de nonconsentement pour les mineurs, témoignent de ces métamorphoses en cours en matière de permis et d'interdit sexuel. Encore inachevées et peu comprises, ces transformations génèrent des tensions et des incertitudes, et posent des questions inédites : un mineur peut-il consentir à un acte sexuel avec un adulte ? La réponse du législateur n'a pas été si claire. L'article 2, définitivement adopté dans la loi n²018 du 3 août 2018, ne retient pas d'âge légal de non-consentement du mineur, mais étend le régime juridique de la contrainte morale. Cette extension risque d'être encore plus difficile à établir, car selon certains pénalistes, le texte reste confus et manque de lisibilité (Darsonville, 2018). Si de telles incertitudes persistent dans la loi, comment affirmer avec force un interdit majeur que l'on énonce mal ?

\section{BIBLIOGRAPHIE}

AMBROISE-RENDU, Anne-Claude. 2014. Histoire de la pédophilie. XIXe-XXIe siècle. Paris : Fayard [ePub].

COSTE, François Louis. 1997. « Le sexe, la loi et le juge ou évolutions d'un principe séparateur instituant l'altérité » Recueil Dalloz : 179.

CROMER, Sylvie. 2017. « Perspective sociologique des viols commis par des majeurs sur des majeurs » AJ Pénal, 255(6) : 260.

CROMER, Sylvie. 2017. « Brève analyse des dossiers judiciaires clos en 2012 dans la juridiction de Lille concernent les violences sexuelles intrafamiliales sur mineur. e. $s$ » In « Les violences sexuelles sur mineur. e. s à caractère incestueux ». Rapport remis à la ministre des Familles, le 26 avril 2017, Expertise, CNRS : 18-20.

DARSONVILLE, Audrey. 2018. «L'article 2 du projet de loi sur les violences sexistes et sexuelles : des avancées, des incertitudes et des craintes pour l'avenir » Le droit en débat [En ligne], consulté le 5/10/18. https://www.dalloz-actualite.fr/chronique/l-article-2-du-projet-de-loi-sur-violencessexistes-et-sexuelles-des-avancees-des-incertit\#.W7ofD3szZOW

DELORS, Germain. 2011. « Le consentement des mineurs victimes d'infractions sexuelles » RSC, 817.

DUMONT, Louis. 1979 [1967]. Homo hierarchicus. Le système des castes et ses implications. Paris : Gallimard. 
GARNOT, Benoit. 2000. «Justice, infrajustice, parajustice et extra justice dans la France d'Ancien Régime » Crime, Histoire \& Sociétés [En ligne], Vol. 4, n 1 |mis en ligne le 02 avril 2009, consulté le 02 octobre 2016. URL : http://chs.revues.org/855 ; DOI : 10.4000/chs.855.

GIULIANNI, Fabienne. 2010. « Le fantasme de l'inceste au prisme de l'écriture des pornographes de la Révolution française » Revue Hypothèses, (13) : 257-265.

GLANDIER-LESCURE, Nathalie. 2006. L'inceste en droit français contemporain. Aix en Provence : Presses Universitaires d'Aix-Marseille.

GRUNVALD, Sylvie. et al. 2016. « Le viol dans la chaîne pénale. Etude à partir des dossiers consultés au TGI de Nantes. Juin 2012 -Septembre 2016 » Mission de recherche Droit et Justice.

GUERY, Christian. 2010. « Définir ou bégayer : la contrainte morale après la loi sur l'inceste » $A J$ pénal : 126 .

HERITIER, Françoise. 1994. Les deux sœurs et leur mère. Paris : Odile Jacob.

KOERING-JOULIN, Renée. 2006. « Brèves remarques sur le défaut de consentement du mineur de 15 ans victime de viols ou d'agressions sexuelles » Mélanges J. Pradel, Editions : Cujes, 389-394.

MAYAUD, Yves. 2004. «Les violences sexuelles, les qualifications relatives aux atteintes sexuelles » Revue AJ Pénal, Janvier (1) : 9-14.

MAYAUD, Yves. 2017. « Le viol : deux lois interprétatives pour une définition! », AJ Pénal : 257.

MONTAS, Arnaud \& ROUSSEL, Gildas. 2010. « La pénalisation explicite de l'inceste : nommer l'innommable » Archives de politique criminelle, 1 (32) : 300.

MUCCHIELLI, Laurent \& LE GOAZIOU, Véronique. 2009. « Contribution à l'analyse de la "violence des mineurs". Les affaires traitées par les juges des enfants » Adolescence, 2 (68) : 415-429. DOI 10.3917/ado.068.0415.

MUCCHIELLI, Laurent \& LE GOAZIOU, Véronique. 2010. « Les viols jugés en Cours d'assises : typologie et variation géographiques » Questions pénales. CESDIP

PORCHY, Marie-Pierre. 2003. Les silences de la loi : un juge face à l'inceste. Paris : Hachette.

ROMERO, Marie. 2017. «Qualifier pénalement l'inceste : les incertitudes du droit pénal français » in La peur de l'inceste, CARUSO, Juliana \& MICHELET Aude, Les Cahiers de l'anthropologie sociale, (éd.). Paris : L'Herne (15), 127-143.

TERRYN, Fabienne. 2013. «L'inceste en droit pénal français » in L'inceste, bilan des savoirs, DUSSY, Dorothée (éd.). Marseille : La Discussion, 57-121.

THERY, Irène. 2002. « Les trois révolutions du consentement : Pour une approche socio anthropologique de la sexualité » Actes du XXXIIIe colloque français de criminologie, Dalloz : 29-51.

VIGARELLO, George. 1998. Histoire du viol : XVIe-XXe siècle. Paris : Seuil.

VRIGNAUX, Dominique. 1994. « Les comptes de l'inceste ordinaire » in De l'inceste HERITIER, Françoise. Paris : Odile Jacob, 129-169.

\section{NOTES}

1. Anne-Claude Ambroise-Rendu. 2014. Histoire de la pédophilie. XIXe-XXIe siècle. Fayard [ePub]. (Introduction) p. 12. 
2. Après la Révolution française, le terme inceste a totalement disparu des premiers codes pénaux $(1791,1810)$; il est demeuré totalement absent dans la législation contemporaine (code pénal de 1994).

3. Comme en Espagne et au Portugal.

4. La convention Lanzarote (STCE $\left.{ }^{\circ} 220\right)$ du Conseil de l'Europe légifère, au niveau européen, sur la protection des enfants contre l'exploitation et les abus sexuels (signée par la France en 2007 et entrée en vigueur en 2010). Dans ce cadre, il a été préconisé de prendre en compte le lien de famille comme un critère constitutif des infractions sexuelles sur mineurs plutôt que comme une circonstance aggravante. Voir le rapport du comité Lanzarote. $10^{\text {ème }}$ réunion. Conseil de l'Europe. T-ES (2015) 01_fr.pp. 5-6.

5. Georges Vigarello. 1998. Histoire du viol: XVIe-XXe siècle. Paris : Seuil. 357 p.

6. Fabienne Giulianni. 2010. Le fantasme de l'inceste au prisme de l'écriture des pornographes de la Révolution française. Revue Hypothèses. Vol 13. pp. 257-265.

7. Lorsque le viol sur un mineur de 15 ans est commis par un ascendant ou une personne ayant autorité, la peine de réclusion passe de 15 à 20 ans, tandis que pour l'agression sexuelle avec les deux mêmes circonstances aggravantes, la peine d'emprisonnement s'élève à 10 ans (au lieu de 7 ans); comme pour l'atteinte sexuelle sur mineur de 15 ans, lorsqu'elle est commise par un ascendant ou une personne ayant autorité, la peine d'emprisonnement est de 10 ans (au lieu de 5 ans).

8. Le droit permet de qualifier d'incestueux le viol, l'agression sexuelle ou l'atteinte sexuelle commis sur un mineur, conformément aux articles 222-31-1 et 227-27-2 du Code pénal, lorsqu'ils sont commis par l'ascendant, le frère ou la sœur, l'oncle ou la tante, le neveu ou la nièce, ou toute personne au sein de la famille qui a autorité de droit ou de fait sur le mineur.

9. Décisions n²011-163 du 16 septembre 2011 et n²011-222 du 17 février 2012.

10. Loi $\mathrm{n}^{\circ} 2016-297 \mathrm{du} 14$ mars 2016 , article $44,1,1^{\circ}$, a et $2^{\circ}, \mathrm{b}$ « [...] Lorsqu'ils sont commis sur la personne d'un mineur par $1^{\circ}$ Un ascendant ; $2^{\circ}$ Un frère, une sœur, un oncle, une tante, un neveu ou une nièce ; $3^{\circ}$ Le conjoint, le concubin d'une des personnes mentionnées aux $1^{\circ}$ et $2^{\circ}$ ou le partenaire lié par un pacte civil de solidarité avec l'une des personnes mentionnées aux mêmes $1^{\circ}$ et $2^{\circ}$, s'il a sur le mineur une autorité de droit ou de fait ».

11. Fabienne Terryn,. 2013. "L'inceste en droit pénal français ». In. Dussy, Dorothée (Coord.). L'inceste, bilan des savoirs. La Discussion. pp. 57-121.

12. Ibid., p 18.

13. «Les violences sexuelles à caractère incestueux sur mineur.e.s ». Rapport remis le 26 avril 2017 à Madame Laurence Rossignol, ministre de la famille, de l'enfance et des droits des femmes, et à Monsieur Thierry Mandon, secrétaire d'état à l'enseignement supérieur et la recherche. $60 \mathrm{p}$. 14. Arnaud Montas, Gildas Roussel. 2010. « La pénalisation explicite de l'inceste : nommer l'innommable », Archives de politique criminelle 2010/1 (n³2), p. 300.

15. Seule exception, dans l'atteinte sexuelle sur mineur de plus de 15 ans par ascendant ou personne ayant autorité, le lien de famille est une composante à part entière de l'infraction. C'est l'infraction la moins sévèrement condamnée puisqu'elle fait encourir seulement 3 ans d'emprisonnement.

16. Article 222-24 du code pénal.

17. Articles 222-29-1 et 222-30 du code pénal.

18. Article $227-26$ du code pénal.

19. L'article 333 de l'ancien code pénal regroupait l'ensemble des circonstances aggravantes du viol, de l'attentat à la pudeur avec ou sans violence. Seul le $\$ 2$ de l'article 331 était à part, l'inceste étant constitutif de l'attentat à la pudeur sans violence sur mineur de plus de 15 ans.

20. Au moment de l'adoption du nouveau code pénal, le sénat avait contesté la séparation des deux catégories d'infractions (agressions sexuelles et atteinte sexuelles) sur le principe qu'un mineur ne pouvait valablement consentir à des relations sexuelles. Il avait considéré que les 
atteintes sexuelles, même sans violence, devaient être de même nature et de même gravité que les agressions sexuelles commises sur des adultes. Mais la commission mixte paritaire a suivi l'avis du gouvernement et de l'assemblée nationale, et a inséré les infractions dans deux chapitres différents du code pénal.

21. Irène Théry, 2002. "Les trois révolutions du consentement: Pour une approche socio anthropologique de la sexualité ». In XXXIIIe colloque français de criminologie. Actes. Dalloz. p 46. 22. Dominique Vrignaux. 1994. «Les comptes de l'inceste ordinaire ». In Françoise Héritier. (dir.). De l'inceste. Odile Jacob. pp. 129-169. ; Marie-Pierre Porchy. 2003. Les silences de la loi : un juge face à l'inceste. Hachette. 178 p.

23. L'article 222-22-1 du code pénal «La contrainte prévue par le premier alinéa de l'article 222-22 peut être physique ou morale. La contrainte morale peut résulter de la différence d'âge existant entre une victime mineure et l'auteur des faits et de l'autorité de droit ou de fait que celui-ci exerce sur cette victime ».

24. Arnaud Montas et Gildas Roussel. 2010, op. cit., p. 297.

25. Cass. Crim. 7 décembre 2005, Bull. crim. $\mathrm{n}^{\circ} 326$.

26. Ibid.

27. Commentaire. Décision $n^{\circ}$ 2014-448 QPC du 6 février 2015. M. Claude A. (Agression sexuelle commise avec une contrainte morale). Conseil constitutionnel. I, A, 2, « La question de la contrainte morale $\gg \mathrm{p} 4$.

28. Affaires de Pontoise en septembre 2017 et Melun en octobre 2017. Dans la première, le parquet n'avait pas retenu la qualification de viol pour les relations sexuelles imposées par un homme de 28 ans à une fille de 12 ans, mais celle d'atteinte sexuelle, considérant qu'il n'y avait eu aucune contrainte, et renvoyé l'affaire devant un tribunal correctionnel. Dans la seconde, un homme de 22 ans, accusé de viol sur une fille de 12 ans, a été acquitté par la Cour d'Assises par manque de preuve de non-consentement.

29. Proposition de loi $n^{\circ} 252$ "Améliorer la protection juridique des mineurs victimes de viol », déposée le 28 septembre 2017, par la députée des Ardennes, Bérengère Poletti (et 37 autres, dont Éric Pauget), et enregistrée à l'Assemblée Nationale le 3 octobre 2017.

30. Article $2,1^{\circ}, \mathrm{b}$ ) « Lorsque les faits sont commis sur la personne d'un mineur e quinze ans, la contrainte morale ou la surprise sont caractérisées par l'abus de vulnérabilité de la victime ne disposant pas du discernement nécessaire pour ces actes ».

31. Article $2, \mathrm{I}, 3^{\circ}$.

32. Article $1, \mathrm{I}, 1^{\circ}$.

33. Sous la direction scientifique de Laurent Mucchielli et le pilotage de Véronique Le Goaziou, deux enquêtes ont été conduites au CESDIP (Centre de Recherches Sociologiques sur le Droit et les Institutions Pénales). La première, intitulée « La violence des mineurs et son traitement pénal : état des lieux et évolutions ", réalisée entre 2007 et 2009 ; La seconde enquête, intitulée " Les déterminants de la criminalité sexuelle. Etude de viol », entre 2009 et 2010.

34. Dans le cadre d'une recherche financée par la Mission Droit et Justice 12-20, deux équipes de recherche ont effectué une étude des dossiers judiciaires de viols et agressions sexuelles auprès du Tribunal de grande Instance (TGI) de Nantes et de Lille sur l'année 2012, intitulée « Le viol dans la chaîne pénale ».

35. Benoit Garnot. 2000. Crimes et Justices aux XVIIe et XVIIIe siècles. Editions Imago. p. 11.

36. Plus de $80 \%$ des affaires sexuelles jugées sont des condamnations pour délits sexuels (agression sexuelle, atteinte sexuelle, exhibitionnisme, corruption de mineur, harcèlement).

37. 6 affaires impliquant uniquement des prévenus mineurs : G3, G26, G28, G30, N28, N29.

38. 15 affaires : N1, N5, N18, N19, N20, N26, G19, G25, G37, N15 (ascendants), G1, G4, N2, N10, G15 (beaux-pères).

39. 7 affaires: G3, G26, G30 (fratrie), N29 (oncle), N4, N25 (cousins), G42 (beau-frère).

40. 5 affaires : G28, N28, N11, G6, N22. 
41. Marie Romero. 2017. «Qualifier pénalement l'inceste : les incertitudes du droit pénal français ", Les Cahiers de l'anthropologie sociale, n¹5, La peur de l'inceste. L'Herne. pp. 127-143.

42. Faute d'alliance, il ne s'agit pas d'un beau-frère et de belle-sœur stricto-sensu. Néanmoins, il existe un lien d'apparentement lié ici au contexte de vie commune : Anthony vit maritalement avec la sœur de la victime.

43. Garde à vue

44. Extrait de l'expertise psychiatrique réalisée sur Anthony. Affaire G42.

45. Extrait PV 2 ème audition de GAV Anthony. Affaire G42.

46. Extrait PV audition de Sophie. Affaire G42.

47. Extrait PV $2^{\text {ème }}$ audition de GAV Anthony. Affaire G42.

48. Ibid., Affaire G42.

49. Extrait des courriers écrits par Anthony, joints au dossier. Affaire G42.

50. Extrait expertise psychologique de Sophie. Affaire G42.

51. Extrait du jugement correctionnel du 20 août 2010, affaire G42.

52. Fichier judiciaire automatisé des auteurs d'infractions sexuelles.

53. Selon l'anthropologue Françoise Héritier, l'inceste de $2^{\text {ème }}$ type correspond aux actes sexuels commis dans un même lieu, en un même temps et pour un même homme entre deux sœurs (ou mère/fille). Voir Françoise Héritier, Les deux sœurs et leur mère. 1994. Odile Jacob. 396 p.

54. Extrait des notes d'audience du 20 août 2010, huit clos. Affaire G42.

55. 16 ans en Espagne, 13 ans en Angleterre, 14 ans en Allemagne. Les législations ne nécessitent pas de retenir, dans l'agression sexuelle, la violence, contrainte, menace ou surprise pour prouver l'absence de consentement du mineur.

56. Cass. Crim. 7 décembre 2005, Bull. crim. $\mathrm{n}^{\circ} 326$.

57. Ibid.

58. Yves Mayaud. 2017. «Le viol : deux lois interprétatives pour une définition! », AJ Pénal. p. 257.

59. 8 affaires : N35, N29, N11, N31, G28, G30, G44, G27.

60. Extrait du soit-transmis du parquet des mineurs. Côte D11. Affaire N20.

61. Extrait de l'audition d'Emilie, côte D33 à D39. Affaire N20.

62. Extrait de l'audition du professeur de Français d' Emilie. Côte D21 à D24 Affaire N20.

63. Extrait de l'audition du confient d'Emilie. Côte D37. Affaire N20.

64. Extrait de l'audition de garde à vue du père. Côte D112 à D114. Affaire N20.

65. Extrait du rapport d'expertise psychiatre de Patrick. Côte 103. Affaire N20.

66. Extrait du courrier du père. Côte D 115. Affaire N20.

67. Extrait du rapport d'expertise psychiatrique. Côte B12. Affaire N20.

68. Ibid., Affaire N20.

69. Ordonnance de Renvoi devant le Tribunal Correctionnel

70. Extrait de l'ORTC. Côte D514-515. Affaire N20.

71. Extrait de l'audition de Léa par officier de police judiciaire, enquête de flagrance. Affaire N15.

72. Extrait du compte rendu de l'assistance à audition de Léa par le psychologue. Affaire N15 (les phrases sont ont été soulignées par l'expert).

73. Ibid. Affaire N15.

74. Extrait de $1^{\text {ère }}$ audition de GAV de Jacques. Affaire N15.

75. Extrait de l'expertise psychiatrique réalisée durant la GAV du grand-père. Affaire N15.

76. Extrait audition de la tante, enquête de flagrance. Affaire N15.

77. Extrait du rapport de synthèse d'enquête préliminaire. Affaire N15.

78. Extrait du compte rendu de jugement correctionnel. Affaire N15.

79. Extrait des notes d'audience de jugement correctionnel. Affaire N15.

80. Il s'agit de la somme la plus élevée dans notre corpus en matière de dommages et intérêts. 
81. Ce préjudice corporel estimé à $30 \%$, porte à la fois sur l'incidence professionnelle, le déficit fonctionnel temporaire, les souffrances endurées jusqu'à présent, le déficit permanent (perte de qualité de vie) et le préjudice esthétique et sexuel.

82. Rappelons que l'atteinte sexuelle sur mineur de plus de 15 ans par ascendant est moins sévèrement réprimée que l'agression sexuelle par ascendant : on passe d'une peine maximale de 3 ans d'emprisonnement à 7 ans.

83. L'atteinte sexuelle sur mineur ne peut être constituée que si l'auteur est un majeur.

84. Par exemple en Autriche, la loi punit tout contact sexuel entre mineurs de moins de 14 ans (âge de la majorité sexuelle) s'il y a un écart d'âge de plus de 4 ans et de plus de 3 ans si c'est une relation sexuelle complète. Site de la « Protection des mineurs en Europe " http:// www.protection-of-minors.eu/fr/cat10.php [Consulté le 21 novembre 2017].

85. Le jeu consistait alors à se déshabiller elle et son frère, et à se mettre nus sous les draps, dans le lit, et à faire semblant de prendre une douche. Son frère était âgé de 7 ans et Sabine de 5 ans.

86. C'est-à-dire issus du même père, mais de mère différente.

87. Extrait de l'audition de plainte de la mère, côte D4, affaire G26.

88. Ibid., côte B3, affaire G26.

89. Extrait audition du père, côte $D 80$, enquête préliminaire, affaire $G 26$.

90. Extrait audition de mère de Jordan, côte D95, enquête préliminaire, affaire G26.

91. Extrait expertise psychologique Jordan, côte B12. Affaire G26

92. Extrait compte-rendu d'enquête de police, affaire G26.

93. Le jeu consistait à se déshabiller Sabine et son frère, à se mettre nus dans le lit, sous les draps, et à faire semblant de prendre une douche. Jordan était âgé de 7 ans, Sabine de 5 ans.

94. Extrait audition de première comparution, Jordan, Côte D120, affaire G26.

95. Ibid., Côte D124, affaire G26.

96. Extrait de la confrontation Jordan/Sabine, côte D185, affaire G26.

97. Extrait audition sur commission rogatoire de la cousine, côte D204, affaire G26.

98. Extrait de l'ORTPE, côte D232. Affaire G26.

99. Extrait du courrier adressé par la mère au procureur, deux mois après la notification de renvoi. Pas de côte. Affaire G26. Il ne s'agit pas ici d'un appel de l'ORTPE.

100. Yves Mayaud, 2004. "Les violences sexuelles, les qualifications relatives aux atteintes sexuelles ». Revue AJ Pénal. Janvier 2004/1. pp. 9-14.

101. Décision n²014-448 QPC du 6 février 2015, 7 : « [...] la seconde phrase de l'article 222-22-1 du code pénal a pour seul objet de désigner certaines circonstances de fait sur lesquelles la juridiction saisie peut se fonder pour apprécier si, en l'espèce, les agissements dénoncés ont été commis avec contrainte; qu'elle n'a en conséquence pas pour objet de définir les éléments constitutifs de l'infraction; qu'il s'ensuit que, dès lors qu'il ne résulte pas de ces dispositions qu'un des éléments constitutifs du viol ou de l'agression sexuelle est, dans le même temps, une circonstance aggravante de ces infractions, ces dispositions ne méconnaissent pas le principe de légalité des délits ».

\section{RÉSUMÉS}

Dans le droit pénal français contemporain, l'inceste est indirectement sanctionné comme circonstance aggravante des infractions sexuelles (viol, agression sexuelle, atteinte sexuelle sur mineur de 15 ans) lorsque l'auteur est un ascendant ou une personne ayant autorité sur la 
victime. S'il n'est plus un crime spécifique, comme dans l'ancien droit, c'est que la loi contemporaine punit avant tout l'atteinte au consentement. Cependant, dans la pratique judiciaire, l'atteinte au consentement s'avère particulièrement complexe et difficile à prouver. Cette question a été au cœur des débats ayant entouré la loi de répression sur l'inceste (2010, 2016) et celle contre les violences sexuelles et sexistes (2018). Aussi, ma contribution propose d'interroger ces évolutions et les problèmes judiciaires liés au traitement pénal de l'inceste. À partir d'un corpus d'affaires collectées à l'occasion de ma thèse portant sur le traitement juridique des délits sexuels sur mineurs, je tenterai, sous l'angle de la sociologie judiciaire, de montrer les changements de normes autour de la question des âges et du consentement.

In the modern French penal code, incest is only considered as an aggravation of criminal offense (rape, sexual offense, indecent assault on minors) when the offender is an ascendant or a person with authority over the victim. If incest is not a specific crime anymore, as in old law, it's because French criminal law punishes first the breach of consent of the person. However, in judicial practices, the non-consent is complex and difficult to prove. This question has been the center of debates on the incest repression law (2010) and the sexual violence and gender-based violence law (2018). My contribution proposes to examine this evolutions and juridical problems related to the penal treatment of incest. My work is based on a legal cases corpus of child sexual abuses, collected for my thesis on juridical treatment of sexual offense on minors. I will try, from a judicial sociology approach, to show the changes of juridical norms on the matter of ages and consent.

\section{INDEX}

Thèmes : Recherches

Keywords : incest, penal qualification, sexual abuse, age, minor, consent

Mots-clés : inceste, qualification pénale, infraction sexuelle, âge, mineur, consentement

\section{AUTEUR}

\section{MARIE ROMERO}

Docteure en sociologie, chercheure correspondante au Centre Norbert Elias de Marseille, Marie Romero travaille sur les représentations sociales et le traitement pénal des violences sexuelles sur mineurs en France (inceste, correctionnalisation du viol, violences sexuelles perpétrées par des mineurs sur d'autres mineurs). Elle a soutenu en février 2018 une thèse à l'EHESS intitulée « Le traitement juridique des délits sexuels sur mineurs, une enquête de sociologie législative et judiciaire » 